Cristian Marchioli · Maria Vittoria Salvetti · Alfredo Soldati

\title{
Appraisal of energy recovering sub-grid scale models for large-eddy simulation of turbulent dispersed flows
}

\author{
Dedicated to Professor Wilhelm Schneider on the occasion of his 70th birthday
}

Received: 10 February 2008 / Revised: 16 May 2008 / Published online: 5 August 2008

(C) Springer-Verlag 2008

\begin{abstract}
Current capabilities of Large-Eddy Simulation (LES) in Eulerian-Lagrangian studies of dispersed flows are limited by the modeling of the Sub-Grid Scale (SGS) turbulence effects on particle dynamics. These effects should be taken into account in order to reproduce accurately the physics of particle dispersion since the LES cut-off filter removes both energy and flow structures from the turbulent flow field. In this paper, we examine the possibility of including explicitly SGS effects by incorporating ad hoc closure models in the Lagrangian equations of particle motion. Specifically, we consider candidate models based on fractal interpolation and approximate deconvolution techniques. Results show that, even when closure models are able to recover the fraction of SGS turbulent kinetic energy for the fluid velocity field (not resolved in LES), prediction of local segregation and, in turn, of near-wall accumulation may still be inaccurate. This failure indicates that reconstructing the correct amount of fluid and particle velocity fluctuations is not enough to reproduce the effect of SGS turbulence on particle near-wall accumulation.
\end{abstract}

\section{Introduction}

Turbulent dispersed flows in boundary layer are found regularly in many industrial and environmental applications involving mixing, combustion, depulveration, spray dynamics, pollutant dispersion and cloud dynamics to name a few. In most applications, the key information is space distribution of particles which is known to be strongly non-homogeneous. Specifically, inertial particles distribute preferentially at the periphery of strong vortical regions and segregate into straining regions [1-4]. Numerical simulations (see [5,6] among others)

It is our great pleasure to take part in this Festschrift Issue dedicated to Professor Wilhelm Schneider on the occasion of his 70th birthday. Happy birthday Willi! We wish you many more fruitful and happy years with the same enthusiasm for science and knowledge you always had.

C. Marchioli · A. Soldati $(\varangle)$

Centro Interdipartimentale di Fluidodinamica e Idraulica and Dipartimento di Energetica e Macchine,

Università degli Studi di Udine, 33100 Udine, Italy

E-mail: soldati@uniud.it

Tel.: +39-(0432)-558020

M. V. Salvetti

Dipartimento di Ingegneria Aerospaziale, Università degli Studi di Pisa, 56100 Pisa, Italy

A. Soldati

Department of Fluid Mechanics, CISM, 33100 Udine, Italy

Present address:

A. Soldati

EPFL, Lausanne, Switzerland 


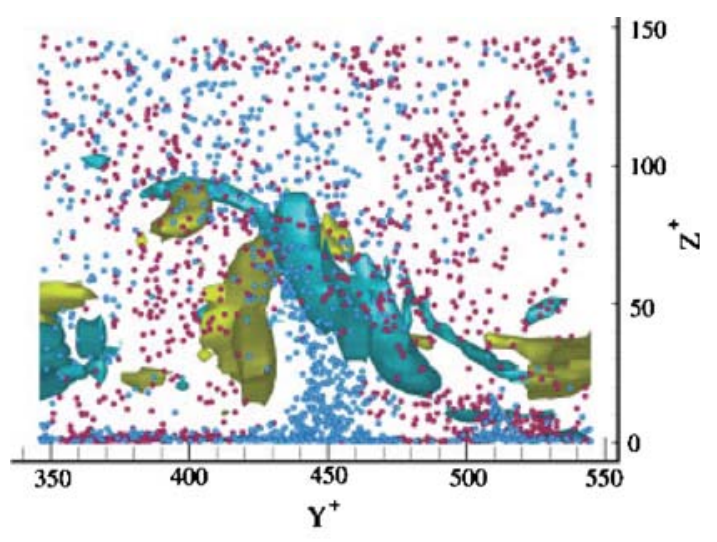

Fig. 1 Front view of particles and structures in the region of particle accumulation (reprint from Soldati [10])

and experiments (see $[7,8]$ among others) have demonstrated that preferential distribution is the result of the interaction between the particles and the vortical coherent structures [9] which control momentum, mass, heat and particle transfer. A snapshot of such interaction is provided in Fig. 1 (from Soldati [10]), obtained from a direct numerical simulation of particle-laden turbulent channel flow at low Reynolds number. Figure 1 shows a cross-sectional view of particles and vortical structures $\left(y^{+}\right.$and $z^{+}$being the dimensionless spanwise and wall-normal coordinates in wall units, respectively). Particles are drawn as circles, larger than the real scale for visualization purposes, and colored according to their wall-normal velocity: blue particles have wall-normal velocity directed away from the wall whereas purple particles have wall-normal velocity directed toward the wall. Vortical structures are rendered using streamwise vorticity isosurfaces: the green isosurface identifies a counter-clockwise rotating quasi-streamwise vortex, whereas the pale blue isosurface identifies a clockwise rotating quasi-streamwise vortex. Pairs of counter-rotating quasi-streamwise vortices are the statistically most common boundary layer coherent structures and are responsible for the flow field examined in Fig. 1 [10]. It is apparent that these vortices are able to entrain particles from the core region toward the wall on their downwash side and away from the wall on their upwash side. In turbulent boundary layers, this transport mechanism, named turbophoresis since the early works by Caporaloni et al. [11] and by Reeks [12], generates net particle fluxes toward the wall leading to irreversible particle accumulation at the wall and to non-uniform particle distribution profiles which peak close to the wall [13]. From a statistical viewpoint, turbophoresis is the outcome of the combined effects of inertial bias between the denser particles and the lighter surrounding fluid $[1,6]$ and the non-homogeneity of the fluid turbulent flow field. In practice, particles deposit at the wall advected down the gradients of fluctuating velocities [12].

Current understanding of particle transport and deposition in turbulent boundary layer indicates that reproducing turbophoresis is crucial to provide reliable predictive models for engineering and/or physical applications [14-18]. In turn, proper modeling of turbophoresis requires accurate characterization of the flow field and of the fluid-particle interaction in the finest scale. In the context of two-phase Eulerian-Lagrangian models, the most common approaches for simulating turbulent fluid flows are: Reynolds-Averaged Navier-Stokes (RANS) equation method, Direct Numerical Simulation (DNS), and Large-Eddy Simulation (LES). RANS methods are formulated primarily for turbulent flows in the core region; therefore, their capability to reproduce the features of turbulence in the near-wall region and to predict quantities such as collision frequency, breakage efficiency, agglomeration, deposition and reaction rates is rather poor, even when ad hoc models for near-wall treatment are used [19]. In spite of this, RANS methods remain as the most commonly used for industrial applications due to their relative simplicity and computational efficiency. DNS-based Eulerian-Lagrangian studies provide the most promising capability to capture turbophoresis by reproducing turbulence effects on particles up to the smallest flow scale (namely, the Kolmogorov scale). Yet the computational requirements associated with current algorithm and computing resources make DNS not applicable to high Reynolds number flows or complex domains of industrial interest. Therefore the use of DNS is limited to investigation of the physics of particle-turbulence interaction in low Reynolds number flows and simple geometries, such as channels $[6,16]$ or pipes [20,21].

Alternative to RANS methods and DNS is LES, where only the large scale motions of the flow are directly simulated whereas the effect of the small, sub-grid scales is modeled. Compared to RANS methods, 

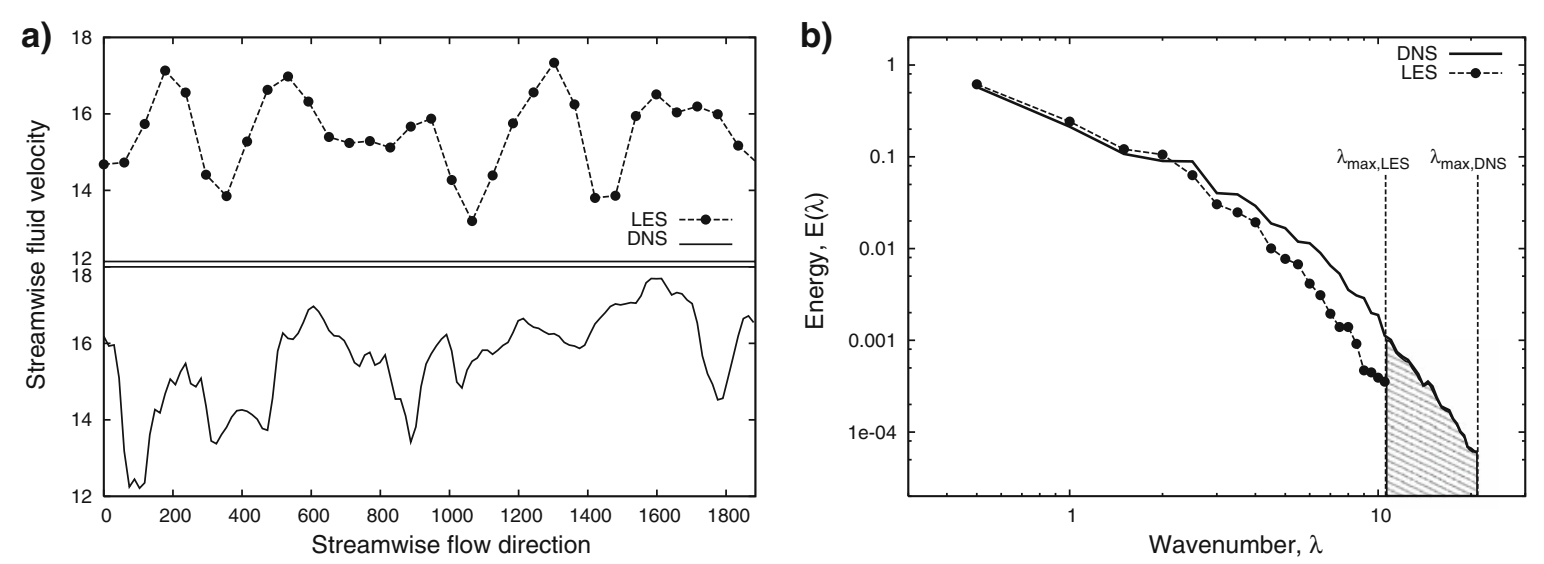

Fig. 2 Comparison between DNS and LES one-dimensional streamwise velocity signals (a) and one-dimensional streamwise energy spectra (b)

LES provides a better representation of turbulence features beyond the sub-grid scales; in addition, it is less demanding than DNS in terms of computational costs. Eulerian-Lagrangian methods based on LES may thus represent a potentially useful tool to predict particle distribution and interaction rates in practical applications where the large flow scales control the particle motion. However, the full capabilities of LES to reproduce segregation phenomena [16,22] and to capture the turbophoretic effect on particle-turbulence statistics [17] are still to be proven. In fact, previous LES applications to the problem of particle dispersion in turbulent bounded flows [14-17,21] have shown that LES tends to underestimate turbophoresis if the effect of the filtered sub-grid scales on particle dynamics is not taken into account [14,21] or properly modeled [15-17]. Marchioli et al. [14], in particular, have shown recently that neglecting the sub-grid turbulence effects in channel flow can lead to significant underestimation of local particle segregation and, in turn, to weaker deposition fluxes and lower near-wall accumulation. Therefore a way to model the effects of the filtered SGS velocity fluctuations on particle motion needs to be identified [14,16,22].

In the present paper, we analyze the problem of incorporating these effects in the Eulerian-Lagrangian modeling of inertial particle dynamics starting from LES channel flow data. Specifically, we examine the possibility to recover the influence of the SGS velocity fluctuations through a closure model for the Lagrangian Particle Tracking (LPT) under the one-way coupling assumption. Candidate closure models tested in this study are based on fractal interpolation $[23,24]$ or on filter inversion by approximate deconvolution $[17,25,26]$. If we compare the rationale of LES to that of DNS, the need to resort to sub-grid closures becomes clear. LES is essentially a coarse-grained filtering of the smaller flow scales and produces a less clearcut rendering of the turbulent flow field (particularly at the finest scales) and a deficit in the turbulent kinetic energy spectra. To elucidate this concept, we can refer to Fig. 2. In this figure one instantaneous realization of the streamwise fluid velocity (Fig. 2a) and the corresponding one-dimensional energy spectrum (Fig. 2b) sampled from DNS and LES of the same flow field considered in Fig. 1 are compared. While the DNS velocity (solid line in Fig. 2a) is characterized by relatively large and sharp variations as well as many fine structures, filtering of smaller flow scales below the cut-off $\Delta$ (corresponding to the wavenumber $\lambda_{\max , \text { LES }}=\pi / \Delta$ in Fig. $2 \mathrm{~b}$ ) produces a LES velocity signal with reduced level of turbulent fluctuations (black circles in Fig. 2a) and narrower spectrum of scales with which the particles can interact. What we aim to assess in this study is the capability of the proposed closure models for LPT to reintroduce the correct amount of SGS turbulent kinetic energy of the flow. In particular, two contributions to the total energy should be recovered to match DNS: The first contribution is given by the energy subtracted from the resolved scales by filtering, corresponding to the gap between the LES spectrum and the DNS spectrum below the $\lambda_{\max }$,LES wavenumber; the second contribution is given by the energy

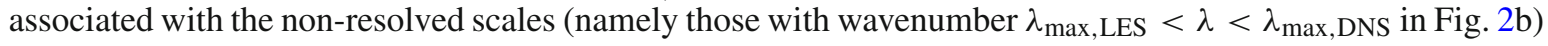
and removed by cut-off, corresponding to the patterned area in Fig. 2b. We remark here that, theoretically, the first contribution can be recovered with both closure models whereas the second contribution can be recovered only with fractal interpolation, that essentially reconstructs the filtered scales of the flow field by extrapolating scales of the coarse-grained LES field to smaller and smaller scales (see Sect. 2.4 for further details).

The paper is organized as follows. Problem statement, governing equations and numerical methodology required for the simulations are presented in Sect. 2. Section 3 is devoted to the analysis and discussion of relevant statistics obtained from simulations where particle trajectories are computed from DNS and LES 
without/with closure model for the particle equations. In this case the discussion will be focused on the quantification of modeling errors on particle velocity and concentration statistics to assess the closure model performance. Final conclusions and possible developments are drawn in Sect. 4.

\section{Physical problem and methodology}

\subsection{Particle-laden turbulent channel flow}

The flow into which particles are introduced is a turbulent channel flow of gas. In the present study, we consider air (assumed to be incompressible and Newtonian) with density $\rho=1.3 \mathrm{~kg} \mathrm{~m}^{-3}$ and kinematic viscosity $v=15.7 \times 10^{-6} \mathrm{~m}^{2} \mathrm{~s}^{-1}$. The governing balance equations for the fluid (in dimensionless form) read as:

$$
\begin{aligned}
& \frac{\partial u_{i}}{\partial x_{i}}=0, \\
& \frac{\partial u_{i}}{\partial t}=-u_{j} \frac{\partial u_{i}}{\partial x_{j}}+\frac{1}{R e} \frac{\partial^{2} u_{i}}{\partial x_{j}^{2}}-\frac{\partial p}{\partial x_{i}}+\delta_{1, i},
\end{aligned}
$$

where $u_{i}$ is the $i$ th component of the dimensionless velocity vector, $p$ is the fluctuating kinematic pressure, $\delta_{1, i}$ is the mean dimensionless pressure gradient that drives the flow and $R e_{\tau}=u_{\tau} h / v$ is the shear Reynolds number based on the shear (or friction) velocity, $u_{\tau}$, and on the half channel height, $h$. The shear velocity is defined as $u_{\tau}=\left(\tau_{w} / \rho\right)^{1 / 2}$, where $\tau_{w}$ is the mean shear stress at the wall. All variables considered in this study are reported in dimensionless form, represented by the superscript + (which has been dropped from Eqs. (1) and (2) for ease of reading) and expressed in wall units. Wall units are obtained combining $u_{\tau}, v$ and $\rho$.

In LES, the standard Continuity and Navier-Stokes equations are smoothed with a filter function of width $\Delta$. Accordingly, all flow variables are decomposed into a resolved (large-scale) part and a residual (sub-grid scale) part as $\mathbf{u}(\mathbf{x}, t)=\overline{\mathbf{u}}(\mathbf{x}, t)+\delta \mathbf{u}(\mathbf{x}, t)$. The filtered Continuity and Navier-Stokes equations for the resolved scales are then:

$$
\begin{aligned}
& \frac{\partial \bar{u}_{i}}{\partial x_{j}}=0, \\
& \frac{\partial \bar{u}_{i}}{\partial t}=-\bar{u}_{j} \frac{\partial \bar{u}_{i}}{\partial x_{j}}+\frac{1}{\operatorname{Re}} \frac{\partial^{2} \bar{u}_{i}}{\partial x_{j}{ }^{2}}-\frac{\partial \bar{p}}{\partial x_{i}}+\delta_{1, i}-\frac{\partial \tau_{i j}}{\partial x_{j}},
\end{aligned}
$$

where $\tau_{i j}=\overline{u_{i} u_{j}}-\bar{u}_{i} \bar{u}_{j}$ represents the sub-grid scale stress tensor. The large-eddy dynamics is closed once a model for $\tau_{i j}$ is provided. In the present study, the dynamic SGS model of Germano et al. [27] has been applied.

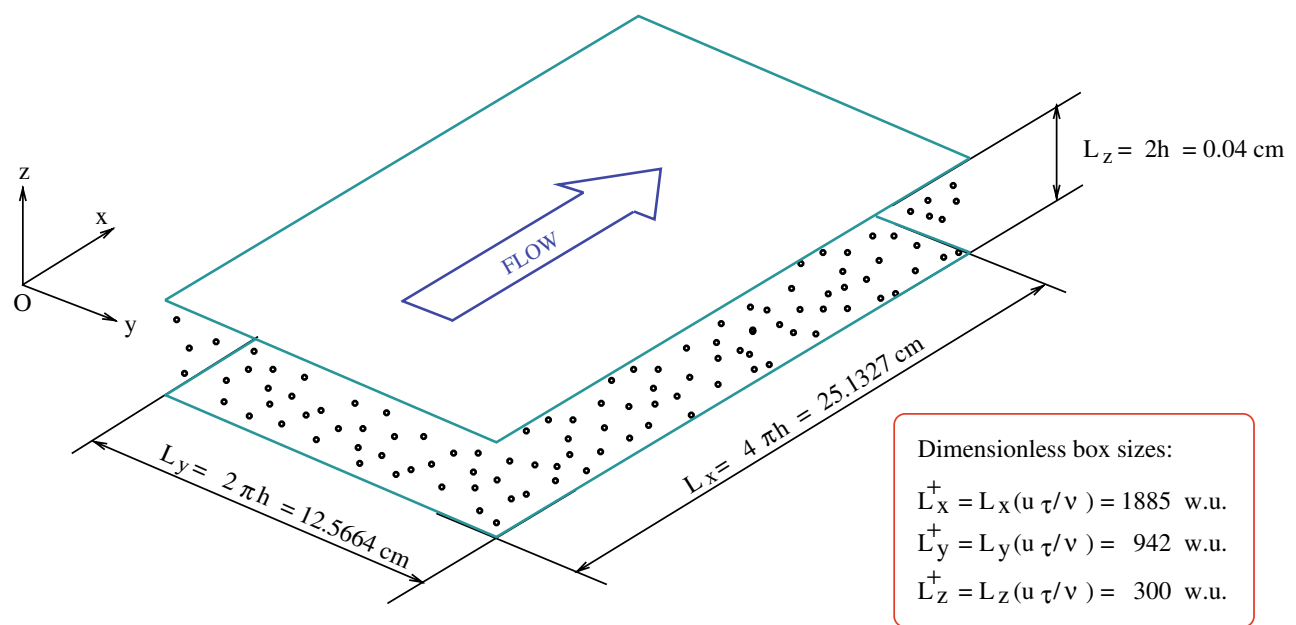

Fig. 3 Particle-laden turbulent gas flow in a flat channel: computational domain 
The reference geometry consists of two infinite flat parallel walls: the origin of the coordinate system is located at the center of the channel and the $x$-, $y$ - and $z$-axes point in the streamwise, spanwise and wallnormal directions respectively (see Fig. 3). Periodic boundary conditions are imposed on the fluid velocity field in $x$ and $y$, no-slip boundary conditions are imposed at the walls. The calculations were performed on a computational domain of size $4 \pi h \times 2 \pi h \times 2 h$ in $x, y$ and $z$, respectively.

Particles with density $\rho_{p}=1000 \mathrm{~kg} \mathrm{~m}^{-3}$ are injected into the flow at concentration low enough to consider dilute system conditions. The motion of particles is described by a set of ordinary differential equations for particle velocity and position. For particles much heavier than the fluid $\left(\rho_{p} / \rho \gg 1\right)$ Elghobashi and Truesdell [28] have shown that the most significant forces are Stokes drag and buoyancy and that Basset force can be neglected being one order of magnitude smaller. In the present simulations, the aim is to minimize the number of degrees of freedom by keeping the simulation setting as simplified as possible; thus the effect of gravity has also been neglected. With the above assumptions, a simplified version of the Basset-Boussinesq-Oseen equation [29] is obtained. In vector form:

$$
\begin{aligned}
& \frac{\mathrm{d} \mathbf{x}}{\mathrm{d} t}=\mathbf{v}, \\
& \frac{\mathrm{d} \mathbf{v}}{\mathrm{d} t}=-\frac{3}{4} \frac{C_{D}}{\mathrm{~d}_{p}}\left(\frac{\rho}{\rho_{p}}\right)|\mathbf{v}-\mathbf{u}|(\mathbf{v}-\mathbf{u}),
\end{aligned}
$$

where $\mathbf{x}$ is particle position, $\mathbf{v}$ is particle velocity, $d_{p}$ is particle diameter and $\mathbf{u}$ is fluid velocity at particle position. The Stokes drag coefficient is computed as $C_{D}=\frac{24}{R e_{p}}\left(1+0.15 R e_{p}^{0.687}\right)$ where $R e_{p}=d_{p}|\mathbf{v}-\mathbf{u}| / \nu$ is the particle Reynolds number. The correction for $C_{D}$ is necessary when $R e_{p}$ does not remain small.

\subsection{DNS and LES methodology}

In this study, both DNS and LES have been applied to the fully-developed channel flow. In both cases, the governing equations are discretized using a pseudo-spectral method based on transforming the field variables into wavenumber space, using Fourier representations for the periodic streamwise and spanwise directions and a Chebychev representation for the wall-normal (non-homogeneous) direction. A two level, explicit AdamsBashforth scheme for the non-linear terms, and an implicit Crank-Nicolson method for the viscous terms are employed for time advancement. Further details of the method have been published previously [30,31].

The value of the shear Reynolds number considered in this study is $R e_{\tau}=150$ based on the shear velocity $u_{\tau}=0.11775 \mathrm{~m} \mathrm{~s}^{-1}$. The corresponding average (bulk) Reynolds number is $R e_{b}=u_{b} h / v=2100$, where $u_{b} \simeq 1.65 \mathrm{~m} \mathrm{~s}^{-1}$ is the average (bulk) velocity. The same value of $R e_{\tau}$ was considered also by Kuerten and co-workers $[16,17]$. We remark here that, at this low $R e_{\tau}$, the $-5 / 3$ extension of the inertial subrange in the energy spectra is not very large (see Fig. 2b, for instance). This is expected to limit the general validity of our results, particularly those obtained using fractal interpolation. The choice of $R e_{\tau}$, however, is imposed by the availability of particle-laden DNS results only at low Reynolds numbers [16].

The size of the computational domain in wall units is $1885 \times 942 \times 300$. The computational domain has been discretized in physical space with $128 \times 128 \times 129$ grid points (corresponding to $128 \times 128$ Fourier modes and to 129 Chebychev coefficients in the wavenumber space). This is the minimum number of grid points required in each direction to ensure that the grid spacing is always smaller than the smallest flow scale and that the limitations imposed by the point-particle approach are satisfied. ${ }^{1}$

LES calculations have been performed on the same computational domain. Two computational grids have been considered: a coarse grid made of $32 \times 32 \times 65$ nodes and a fine grid made of $64 \times 64 \times 65$ nodes.

\subsection{Lagrangian particle tracking}

To calculate particle trajectories in the flow field, we have coupled a Lagrangian tracking routine with the DNS/LES flow solver. The routine solves for Eqs. (6) and (5) using sixth-order Lagrangian polynomials to

\footnotetext{
${ }^{1}$ In the present flow configuration, the dimensionless Kolmogorov length scale, $\eta_{K}^{+}$, varies along the wall-normal direction from a minimum value $\eta_{K}^{+}=1.6$ at the wall to a maximum value $\eta_{K}^{+}=3.6$ at the centerline [32]. The grid resolution in the wall-normal direction is such that the first collocation point is at $z^{+}=0.05$ from the wall, while in the center of the channel $\Delta z^{+}=3.7$.
} 
Table 1 Particle simulation parameters

\begin{tabular}{llllll}
\hline St & $\tau_{p}(\mathrm{~s})$ & $d_{p}^{+}$ & $d_{p}(\mu \mathrm{m})$ & $V_{s}^{+}=g^{+} \cdot S t$ & $R e_{p}^{+}=V_{s}^{+} \cdot d_{p}^{+} / \nu^{+}$ \\
\hline 1 & $1.133 \times 10^{-3}$ & 0.153 & 20.4 & 0.0943 & 0.01443 \\
5 & $5.660 \times 10^{-3}$ & 0.342 & 45.6 & 0.4717 & 0.16132 \\
25 & $28.32 \times 10^{-3}$ & 0.765 & 102.0 & 2.3584 & 1.80418 \\
\hline
\end{tabular}

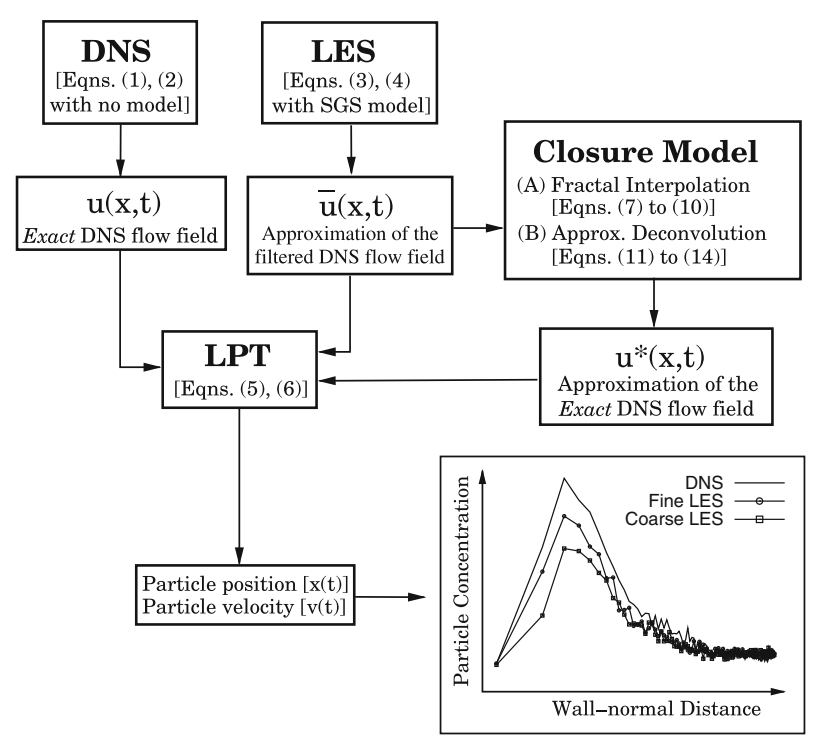

Fig. 4 Summary of the numerical methodology used in this work to study the problem of turbulent particle dispersion

interpolate fluid velocities at particle position; with this velocity the equations of particle motion are advanced in time using a fourth-order Runge-Kutta scheme. The timestep size used for particle tracking was chosen to be equal to the timestep size used for the fluid, $\delta t^{+}=0.045$; the total tracking time was, for each particle set, $t^{+}=1350$. These simulation times are not long enough to achieve a statistically steady state for the particle concentration [33].

Particles, which are assumed pointwise, rigid and spherical, are injected into the flow at concentration low enough to neglect particle collisions. The effect of particles onto the turbulent field is also neglected (one-way coupling assumption). At the beginning of the simulation, particles are distributed randomly over the computational domain and their initial velocity is set equal to that of the fluid at the particle initial position. Periodic boundary conditions are imposed on particles moving outside the computational domain in the homogeneous directions, perfectly elastic collisions at the smooth walls were assumed when the particle center was at a distance lower than one particle radius from the wall.

For the simulations presented here, large samples of $10^{5}$ particles, characterized by different response times, were considered. The response time is defined as $\tau_{p}=\rho_{p} d_{p}^{2} / 18 \mu$ where $\mu$ is the fluid dynamic viscosity: when the particle response time is made dimensionless using wall variables, the Stokes number for each particle set is obtained as $S t=\tau_{p}^{+}=\tau_{p} / \tau_{f}$ where $\tau_{f}=v / u_{\tau}^{2}$ is the viscous timescale of the flow. Table 1 shows all the parameters of the particles injected into the flow field.

\subsection{Closure models for Lagrangian particle tracking}

In the following, the candidate closure models incorporated in the particle equation of motion (Eq. 6) to recover the effect of the filtered SGS turbulent kinetic energy for the fluid velocity field are briefly outlined. This will complete the description of the methodology used in this study, which is summarized in Fig. 4. The reference case is DNS, where the governing equations for the fluid are solved without modeling and thus an exact velocity field, $\mathbf{u}(\mathbf{x}, t)$, is supplied to the LPT equations. The application to be improved is standard LES with no closure model for the LPT equations. In this case, filtered governing equations for the fluid are solved. The result is 
only an approximation, $\overline{\mathbf{u}}(\mathbf{x}, t)$, of an equally filtered DNS flow field, mainly because of modeling error in the closure of the LES equations for the fluid phase. This approximate velocity, however, may not warrant accurate prediction of crucial dispersion phenomena such as particle segregation and accumulation at the wall. A possible way to overcome inaccuracies is the inclusion of an ad hoc closure model capable of yielding an approximation, $\mathbf{u}^{*}(\mathbf{x}, t)$, of the exact DNS field when fed with $\overline{\mathbf{u}}(\mathbf{x}, t)$ as input. Solving for the LPT equations with $\mathbf{u}^{*}(\mathbf{x}, t)$, rather than with $\overline{\mathbf{u}}(\mathbf{x}, t)$, should improve the results: this is precisely what we wish to assess.

\subsubsection{Fractal interpolation}

The aim of fractal interpolation is to reconstruct the velocity field $\mathbf{u}(\mathbf{x}, t)$ from the filtered field $\overline{\mathbf{u}}$. This is done by iteratively applying an affine mapping procedure to $\overline{\mathbf{u}}$. In this way, starting from a coarse grid on which $\overline{\mathbf{u}}$ is defined, a signal can be reconstructed on a given finer grid (we refer to [24,34] for more details). To elaborate, let us consider for simplicity a $1 \mathrm{D}$ case where the dimensionless coordinate $\xi$ is introduced such that $\xi=\left(x-x_{i-1}\right) / 2 \Delta$ in the interval $\left[x_{i-1}, x_{i+1}\right]$. Considering the space $C[\hat{0}, 1]=\{u \in C[0,1] \mid u(0)=$ $\left.\bar{u}_{i-1}, u(1)=\bar{u}_{i+1}\right\}$, the map $W_{i}$ is defined as follows:

$$
\begin{aligned}
& W_{i}[u](\xi)=d_{1} \cdot \bar{u}(2 \cdot \xi)+q_{i, 1}(2 \cdot \xi), \\
& W_{i}[u](\xi)=d_{2} \cdot \bar{u}(2 \cdot \xi-1)+q_{i, 2}(2 \cdot \xi-1),
\end{aligned}
$$

Eq. (7) holds for $\xi \in\left[0, \frac{1}{2}\right]$, while Eq. (8) for $\xi \in\left[\frac{1}{2}, 1\right]$ and:

$$
\begin{aligned}
& q_{i, 1}(\xi)=\left[\bar{u}_{i}-\bar{u}_{i-1}-d_{1} \cdot\left(\bar{u}_{i+1}-\bar{u}_{i-1}\right)\right] \cdot \xi+\bar{u}_{i-1} \cdot\left(1-d_{1}\right), \\
& q_{i, 2}(\xi)=\left[\bar{u}_{i+1}-\bar{u}_{i}-d_{2} \cdot\left(\bar{u}_{i+1}-\bar{u}_{i-1}\right)\right] \cdot \xi+\bar{u}_{i}-d_{2} \cdot \bar{u}_{i-1} .
\end{aligned}
$$

Starting from the LES-like grid, a signal can be reconstructed on a finer DNS-like grid by iteratively repeating this procedure. The characteristics of the reconstructed signal depend on the two stretching parameters, $d_{1}$ and $d_{2}$. It can be shown [24] that these parameters are related to the fractal dimension of the signal. Scotti and Meneveau [24] considered these parameters constant in time and space and the values they adopted are obtained from experimental velocity signals of homogeneous and isotropic turbulence; in particular, these parameters were set to $d_{1}=2^{-1 / 3}$ and $d_{2}=-2^{-1 / 3}$, corresponding to a fractal dimension of the velocity signal of 1.7. In our study, we have applied an algorithm [23] which allows the stretching parameters to be computed locally using the discrete values on a fine grid (DNS data), without the a-priori knowledge of the fractal dimension of the velocity signal. However, the locally-computed values show significant fluctuations reflecting the instantaneous fluctuations of the DNS velocity fields from which they have been derived. Thus, to use these local values in the fractal interpolation procedure, a 3D distribution of the stretching parameters should be computed and stored for each velocity component at each time step. Besides making the procedure much more complex, this computation can not be carried out in actual LES simulations for which the instantaneous DNS fields are obviously not available. To overcome this problem, we adopted the following strategy: from the locally computed values of the stretching parameters we compute the fractal dimension (for each velocity component and at each time step) for fractal interpolation in the $x$ direction, by considering the $x$ distribution of the variable at each discrete location on the horizontal $y-z$ planes as a single signal. Then, the fractal dimension is averaged over the spanwise direction $y$ and in time. The averaged fractal dimension for fractal interpolation in the $y$ direction is computed analogously. The output of this computation is the averaged fractal dimension of each velocity component and for each horizontal plane (constant $z$ ), for reconstruction in the $x$ and $y$ directions, respectively. The corresponding stretching parameters for each velocity component and for each direction ( $x$ and $y$ ) are shown in Fig. 5 as a function of the distance from the wall, expressed here as $z / h$. The values of these parameters are assumed constant in time and over the horizontal planes and are derived from the already-available fractal dimension, using the same relationship as in [24]. Note that in all cases the new stretching parameters show a noticeable variation in the wall-normal direction and they are significantly lower than the value experimentally obtained for homogeneous turbulence. 

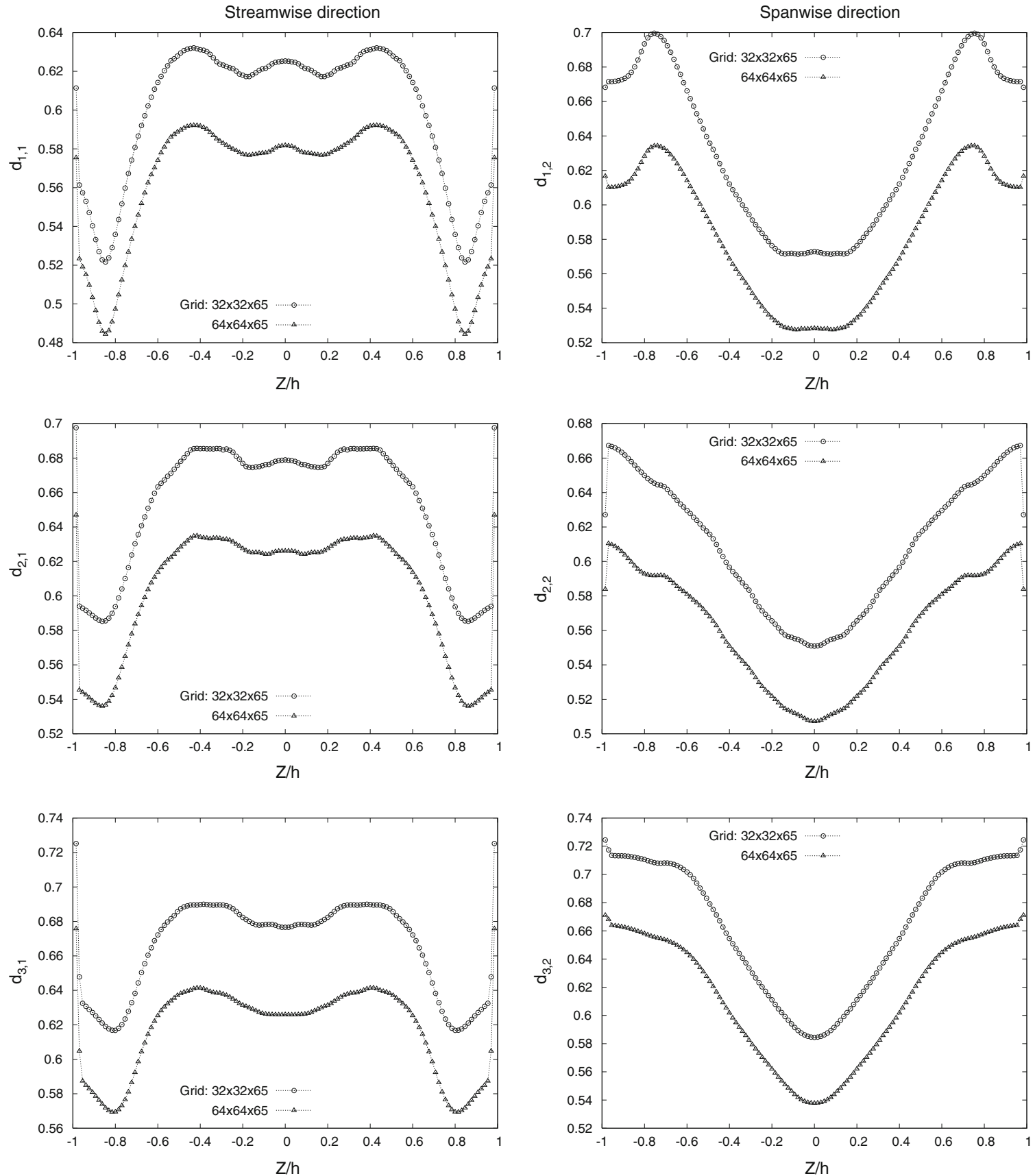

Fig. 5 Wall-normal profiles of the absolute value of the local stretching parameters used to reconstruct the velocity components: $d_{i, 1}$ and $d_{i, 2}$ are the parameters used in fractal interpolation of the $i$ th velocity component in the streamwise direction and in the spanwise direction, respectively. For each horizontal plane, $d_{1}$ in Eqs. (7), (8), (9) and (10) takes the corresponding values in the figures while $d_{2}=-d_{1}$. Symbols: triangles fine LES, circles coarse LES

\subsubsection{Approximate deconvolution}

The filtered velocity, $\bar{u}_{i}(\mathbf{x}, t)$, is defined by the convolution product of the instantaneous velocity, $u_{i}(\mathbf{x}, t)$, and a filter kernel $G$ as

$$
\bar{u}_{i}(\mathbf{x}, t)=G(\mathbf{x} ; \Delta) * u_{i}(\mathbf{x}, t)=\int_{-\infty}^{\infty} u_{i}(\mathbf{y}, t) G(\mathbf{x} ; \mathbf{y} ; \Delta) \mathrm{d}^{3} y,
$$



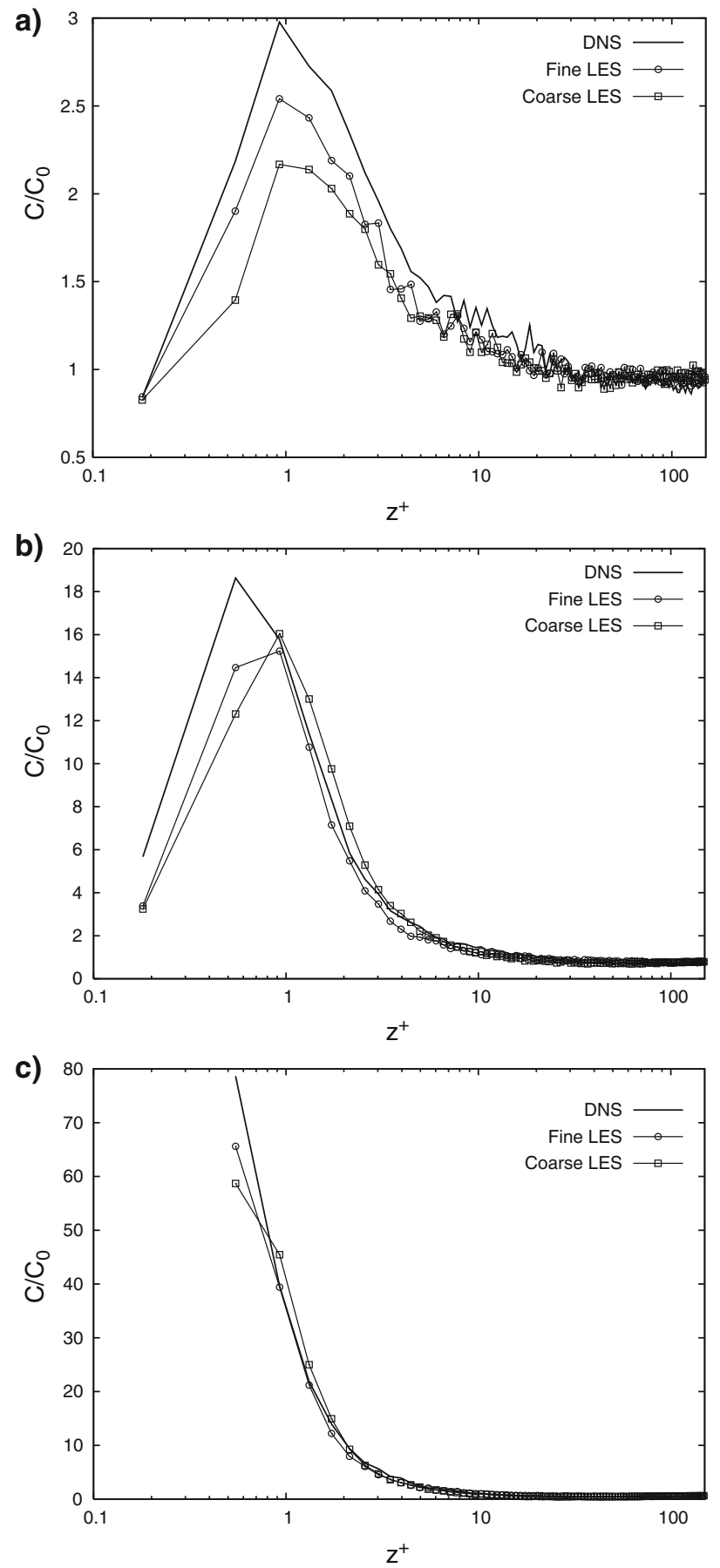

Fig. 6 Instantaneous particle number density distribution: comparison between DNS (solid line) and LES (symbols). Panels: a $S t=1 ; \mathbf{b} S t=5 ; \mathbf{c} S t=25$

where $\Delta$ is the filter size associated with $G$. Assuming that $G$ has an inverse, $G^{-1}$, the inverse operator can be expanded as an infinite series of filter operators. Filters with compact transfer functions are non-invertible, but a regularized inverse operator can be approximated by truncating the series at some $N$ [35]:

$$
G^{-1} \simeq \sum_{\alpha=0}^{N}(I-G)^{\alpha},
$$



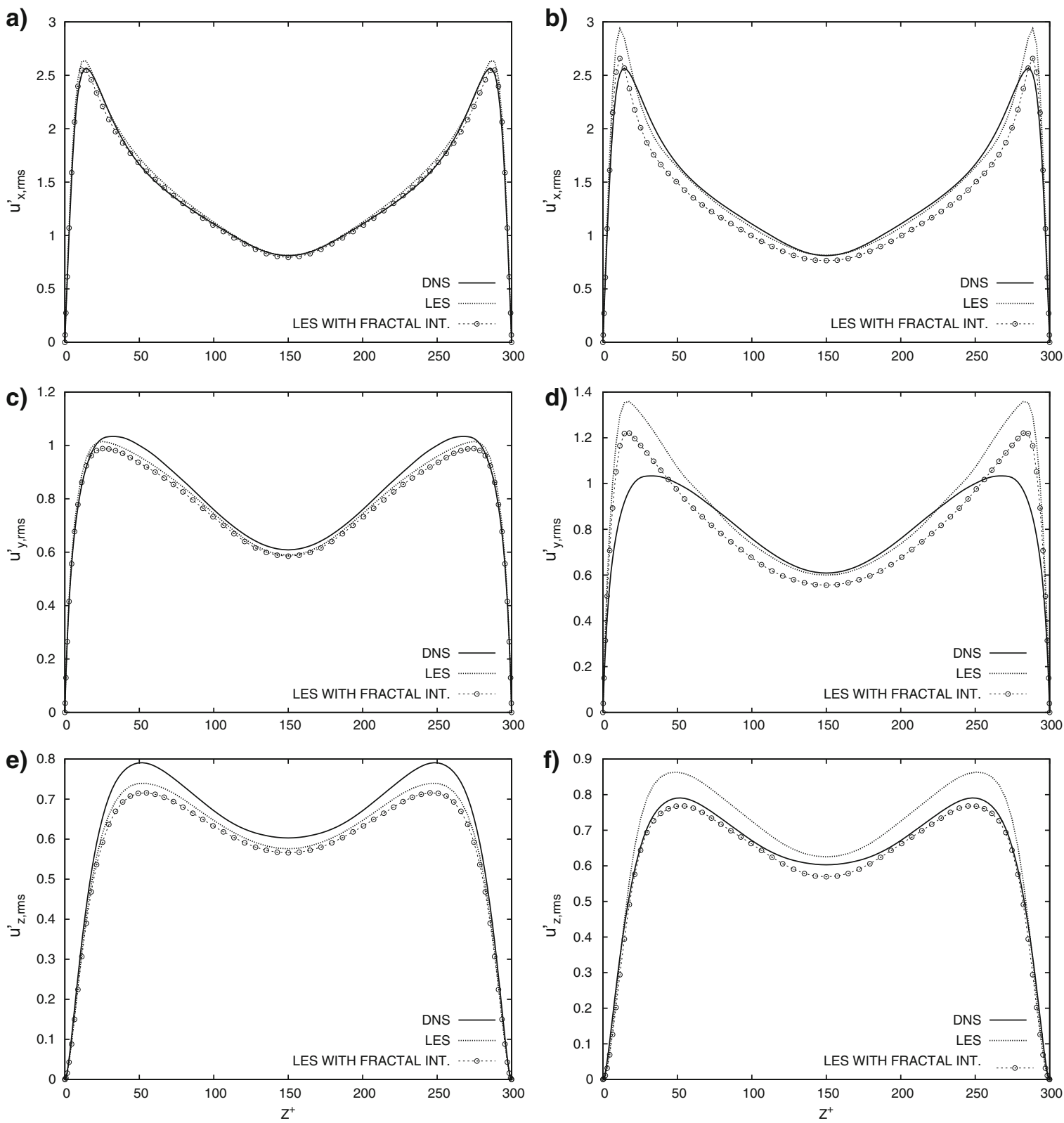

Fig. 7 RMS of fluid velocity fluctuations: comparison between DNS (solid line), LES (dotted line) and LES with closure model for the particles based on fractal interpolation (circles). Panels: a, b streamwise RMS; c, $\mathbf{d}$ spanwise RMS; e, $\mathbf{f}$ wall-normal RMS. Left-hand panels are relative to the fine LES; right-hand panels are relative to the coarse LES

where $I$ is the identity operator and $N$ is the truncation parameter to be fixed: following Stolz et al. [35], we set $N=5$. Using this equation, the approximate deconvolved velocities, $u_{i}^{*}$, can be obtained by consecutively applying the filter [25]:

$$
u_{i}^{*}=\sum_{\alpha=0}^{N}(1-G)^{\alpha} * \bar{u}_{i}
$$

Although the filter implicitly applied by the numerical discretization in the homogeneous directions is the Fourier cut-off filter, the deconvolution procedure requires a smooth filter to be effective (see, e.g., [35]). Among the possible analytic and discrete smooth filters, a top-hat filter was applied in this work, as done also 

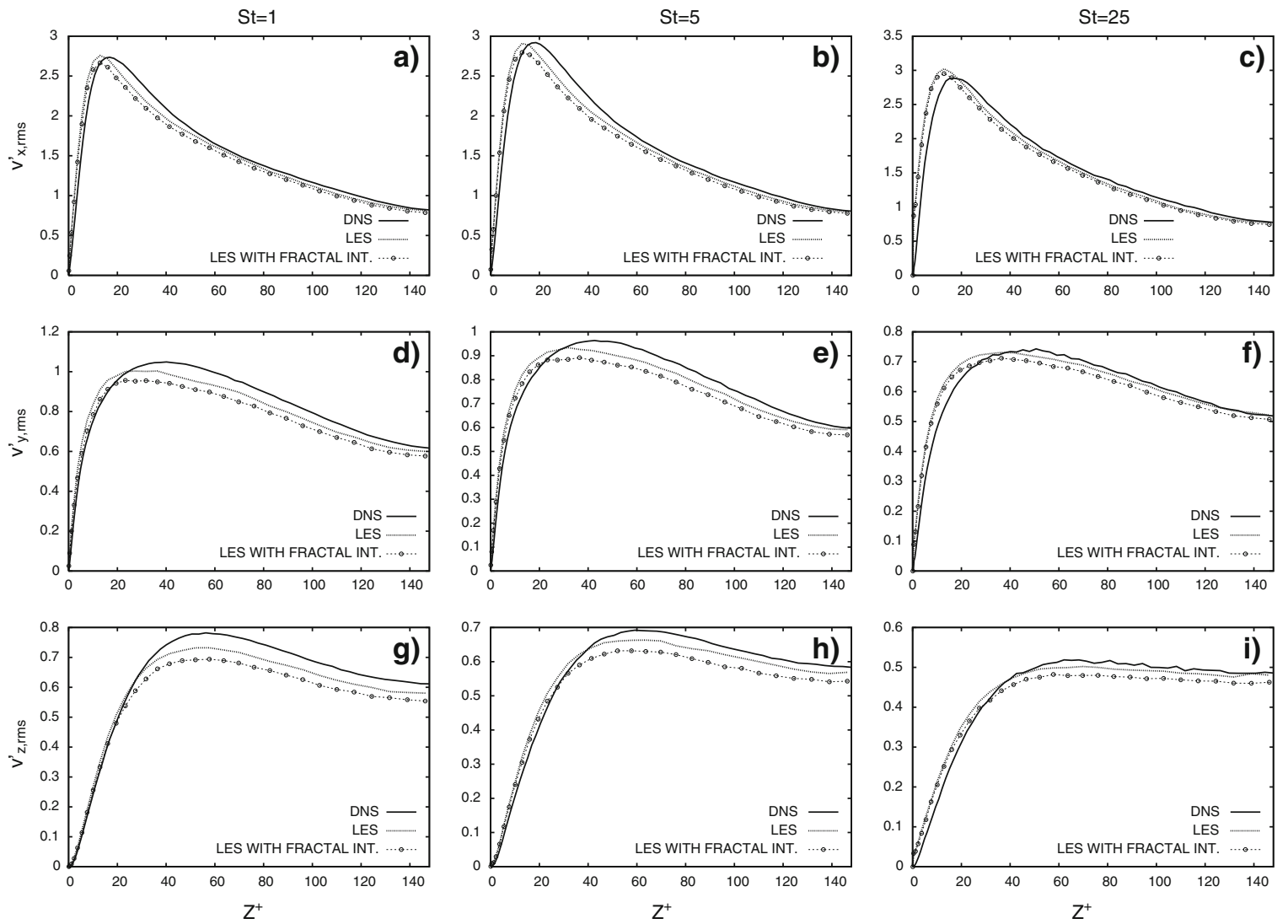

Fig. 8 RMS of particle velocity fluctuations: comparison between DNS (solid line), fine LES (dotted line) and fine LES with closure model for the particles based on fractal interpolation (circles). Panels: a-c streamwise RMS; d-f spanwise RMS; g-i wall-normal RMS. Columns: $S t=1$ (left); St $=5$ (center); St $=25$ (right)

in [17] for instance. In Fourier space, the top-hat filter is defined as follows:

$$
\hat{G}(\lambda)= \begin{cases}\sin (\lambda \Delta / 2) & \text { if } \lambda<\lambda_{\text {cut }- \text { off }} \\ 0 & \text { if } \lambda>\lambda_{\text {cut }- \text { off }}\end{cases}
$$

where $\lambda$ is the wavenumber and $\lambda_{\text {cut }- \text { off }}=\pi / \Delta$.

\section{Results and discussion}

In this section, our main objective is to assess the performance of the candidate energy-recovering closure models by comparing fluid and particle velocity statistics as well as particle concentrations obtained from three different numerical simulations: DNS, LES without SGS closure model for the particle phase (referred to as standard LES hereinafter) and LES with closure model. First, we will discuss briefly the results obtained for standard LES, since these represent the starting point that motivates our study.

\subsection{Results with no SGS closure model for the particle phase}

In Sect. 1, we mentioned that previous LES applications to the problem of particle dispersion in turbulent wall-bounded flows have provided evidence of significant underestimation of turbophoresis and near-wall accumulation when the effect of the SGS fluid velocity fluctuations is neglected or not properly modeled. This tendency is illustrated in Fig. 6, where different instantaneous particle concentration profiles (taken at time $t^{+}=1350$ of the simulations) in the wall-normal direction are compared. Concentration is computed here as particle number density distribution, $C$, normalized by its initial value, $C_{0}$ (see [33] for details). The solid line 

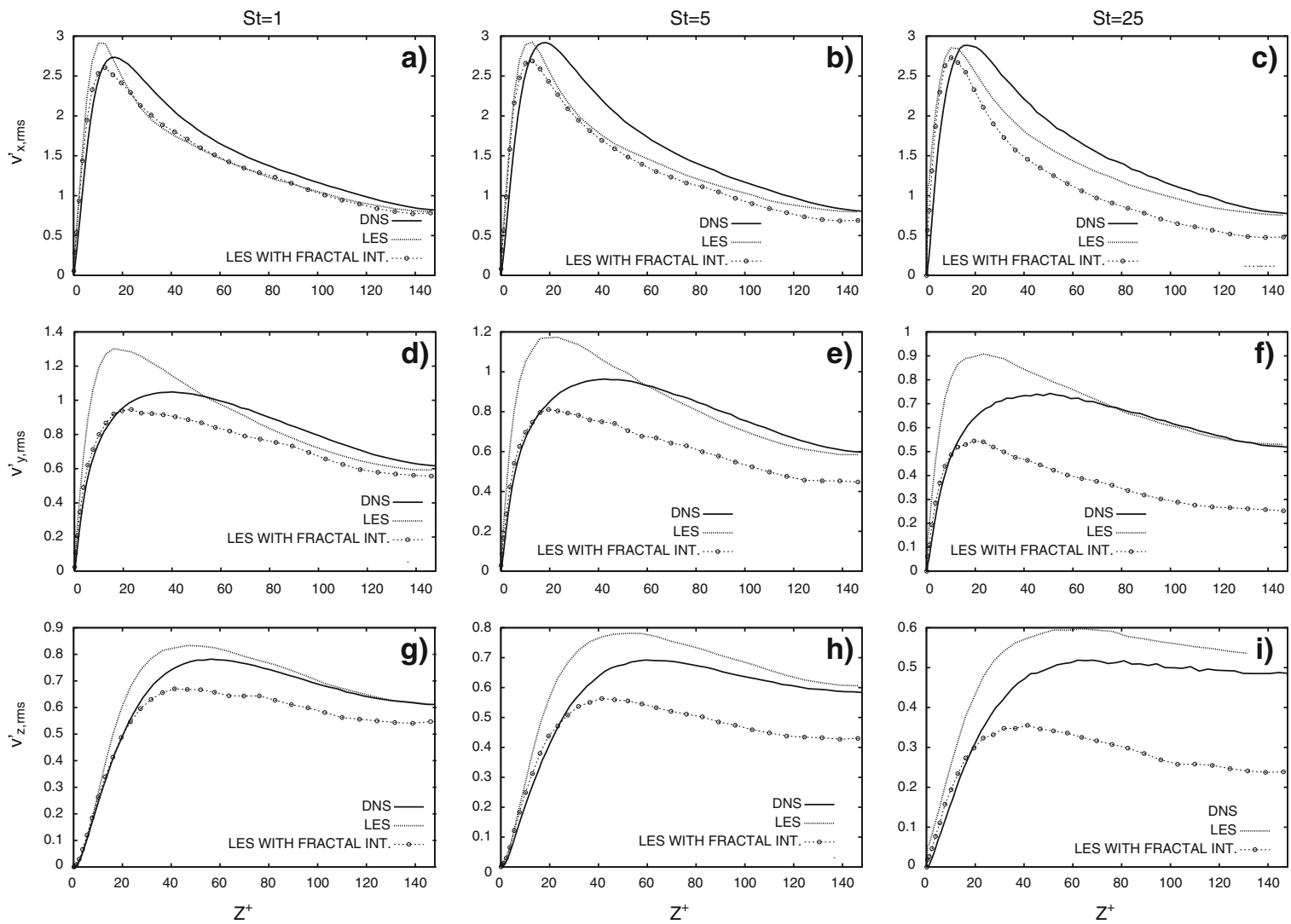

Fig. 9 RMS of particle velocity fluctuations: comparison between DNS (solid line), coarse LES (dotted line) and coarse LES with closure model for the particles based on fractal interpolation (circles). Panels: a-c streamwise RMS; d-f spanwise RMS; g-i wall-normal RMS. Columns: St $=1$ (left); St $=5$ (center); St $=25$ (right)

in each panel of Fig. 6 corresponds to the reference DNS profile; symbols correspond to the fine and the coarse standard LES. All Stokes numbers considered in this study are shown: $S t=1$ (Fig. 6a), $S t=5$ (Fig. 6b) and $S t=25$ (Fig. 6c).

It is apparent that, for the entire range of particles considered, LES underpredicts both qualitatively and quantitatively the turbophoretic effect on particle accumulation at the wall by providing different-shape profiles and lower peaks of particle concentration inside the viscous sublayer. Note that the degree of underprediction also depends on the spatial resolution of the Eulerian grid. The results shown in Fig. 6 can be explained considering that LES filtering has an effect on particle motion which depends on the ratio of the particle size to the filtered spatial scales. As shown recently by Fede and Simonin [22] and by Marchioli et al. [14], particle accumulation is strongly influenced by sub-grid fluid turbulence even when the particle response time is much larger than the flow timescales not resolved in LES (see the $S t=25$ particles, for instance). The inaccuracy of LES in predicting near-wall accumulation is associated to limits in reproducing the sub-grid turbulence effects (through the SGS model for the fluid), which lead to inadequate representation of local segregation phenomena in the buffer region of the turbulent boundary layer. It is precisely for this reason that closure models supplying the particle motion equations with an adequate rendering of the flow field might be needed.

Starting from these observations, in the following we will test the candidate closure models in a systematic way, covering a large target parameter space: three different DNS/LES flow data sets, two closure models and three sets of particles characterized by different inertia, for a total of 15 cases.

\subsection{Results with SGS closure model based on fractal interpolation}

In this section, we will discuss fluid and particle velocity statistics and particle concentration statistics obtained from LES when a closure model for the SGS fluid velocity fluctuations based on 2D fractal interpolation is 

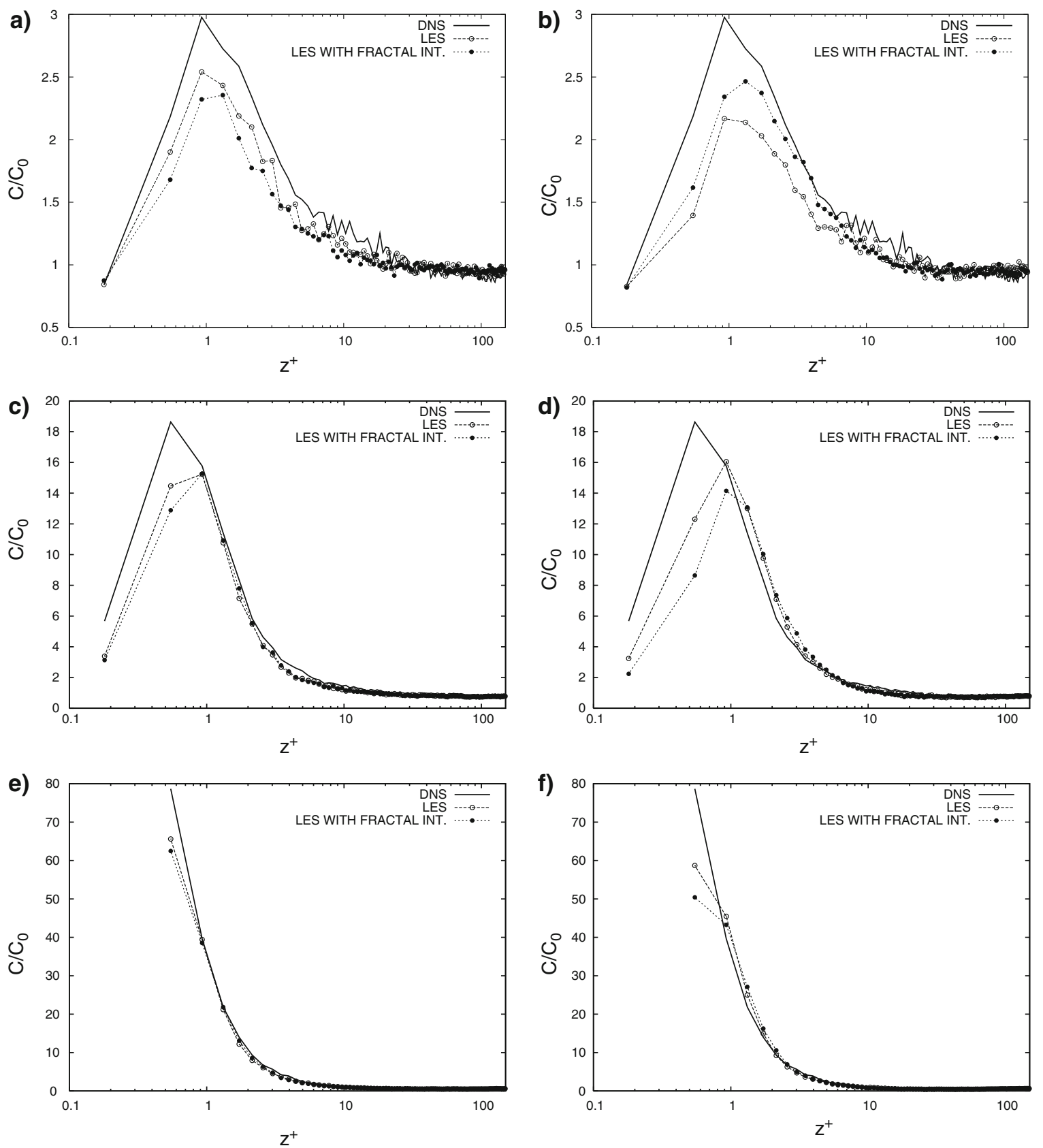

Fig. 10 Effect of the closure model on particle concentration: comparison between DNS (solid line), fine LES (open circles) and fine LES with closure model based on fractal interpolation (black circles). Panels: a, b $S t=1 ; \mathbf{c}, \mathbf{d} S t=5 ; \mathbf{e}, \mathbf{f} S t=25$. Left-hand panels are relative to the fine LES; right-hand panels are relative to the coarse LES

used. In this case, the Eulerian velocity components provided by LES are reconstructed along the homogeneous directions (but not in the wall-normal direction) from the fine/coarse LES grid to the DNS grid in an effort to retrieve part of the sub-grid contributions: these fractally interpolated velocities are then plugged into the particle equation of motion to perform the Lagrangian tracking and, in turn, to compute the statistics.

Figure 7 shows the effect of using fractal interpolation on the components of the Root Mean Square (RMS) of fluid velocity fluctuations, $u_{i, \mathrm{rms}}^{\prime}$. Vís-a-vís comparison is made between DNS (solid line), standard LES (dotted line) and LES with fractal interpolation (open circles) for both LES grids. All profiles were obtained averaging in time (over a time span of 1350 wall units) and space (over the homogeneous directions). Results for the fine LES (Fig. 7a, c, e) indicate that standard LES neglecting the sub-grid scale terms in the particle 


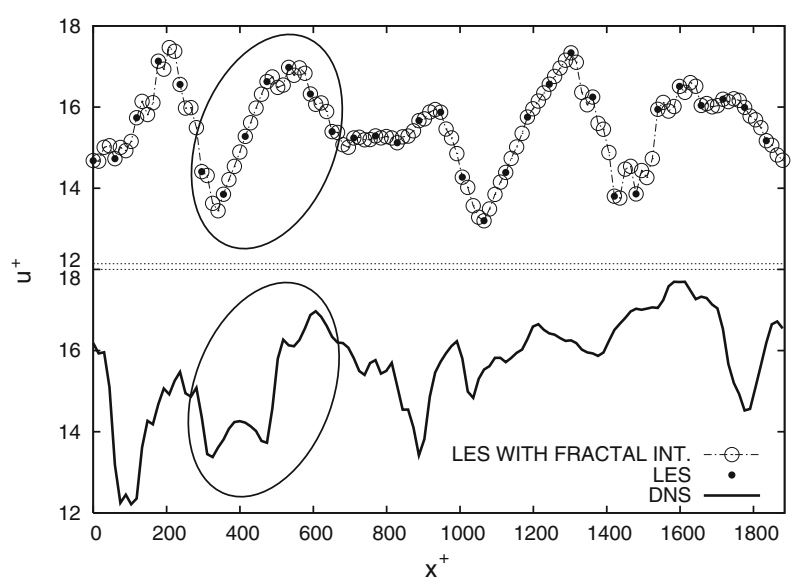

Fig. 11 Turbulent streamwise fluid velocity signal: comparison between DNS (solid line), LES (black circles) and LES with fractal interpolation (open circles)

equation of motion slightly overestimates the streamwise fluid velocity fluctuations $\left(u_{x, \mathrm{rms}}^{\prime}\right)$ with respect to the reference DNS values while it underestimates the spanwise $\left(u_{y, \mathrm{rms}}^{\prime}\right)$ and the wall-normal $\left(u_{z, \mathrm{rms}}^{\prime}\right)$ fluctuations. When fractal interpolation is used to reconstruct the fluid velocity signal, the DNS profile is recovered for $u_{x, \mathrm{rms}}^{\prime}$ whereas a further slight suppression is observed for both $u_{y, \mathrm{rms}}^{\prime}$ and $u_{z, \mathrm{rms}}^{\prime}$. Results for the coarse LES (Fig. 7b, d, f) show an overshoot of all RMS components in standard LES. This may be surprising, since LES is expected to give a filtered field, i.e. a field from which part of the fluctuations (mainly the highest frequency ones) has been removed through filtering. However, the observed behavior is due to the SGS modeling error in the LES equations for the fluid phase, which in the coarser case leads to an overshoot of the RMS of the fluid velocity components. This overshoot is a rather well-known behavior of coarse LES, especially for the RMS of the streamwise component. Due to the reduction of fluid velocity fluctuations when fractal interpolation is used, the wall-normal RMS profile almost collapses on the DNS one. The behavior of the wall-normal RMS component is of particular interest since it has direct impact on turbophoresis, at least for small particle relaxation times: as shown by Kuerten and Vreman [17], the intensity of the turbophoretic drift can be related to the wall-normal velocity fluctuations near the wall and, in turn, to the effect of the filtering made in standard LES on these fluctuations. Thus, the good agreement between the coarse LES with fractal interpolation and the DNS observed in Fig. $7 \mathrm{f}$ is expected to yield improved prediction of the turbophoretic drift with respect to standard LES.

In Figs. 8 and 9, the RMS components of the particle velocity fluctuations, $v_{i, \mathrm{rms}}^{\prime}$, are shown for the fine and for the coarse LES grids, respectively. The reference DNS quantities are also reported. Lines and symbols are as in Fig. 7. All profiles were obtained averaging in time (starting from $t^{+}=450$ after particle initial release in the flow up to $t^{+}=1350$ ) and space (over the homogeneous directions). These statistics bring out the effect on the particles fluctuating velocity field due to use of fractal interpolation. For the fine LES case, standard LES is already in rather good agreement with DNS even when the SGS effects are neglected; the only noticeable modification produced by fractal interpolation is a further damping of all RMS components. The underestimation of particle velocity fluctuations occurs also in the coarse LES case and is much more evident, in particular for the spanwise and wall-normal components of the $S t=25$ particles (Fig. 9f, i, respectively). The large disagreement with DNS is expected to correspond to large inaccuracies in the evaluation of turbophoresis on particle wall accumulation.

To corroborate this conclusion, in Fig. 10 the instantaneous particle concentration profiles for all the considered particle sets are shown. These profiles were computed at the same time of those reported in Fig. 6 . Compared to standard LES, LES with closure model for LPT based on fractal interpolation predicts lower particle wall accumulation build-up rates in all cases under investigation but for the $S t=1$ particles in the coarse grid (this result being in agreement with that of Fig. 7f). Also the particle deposition velocity ${ }^{2}$ is generally underestimated (results are not shown here for brevity).

2 The deposition velocity is one of the most interesting statistics for engineering purposes because it quantifies the rate at which particles deposit at the wall. This velocity can be computed by taking the ratio between the mass flux of particles per unit deposition area, in $\left(\mathrm{kg} \mathrm{s}^{-1} \mathrm{~m}^{-2}\right)$, and the bulk mass concentration of particles, in $\left(\mathrm{kg} \mathrm{m}^{-3}\right)$ [36]. 

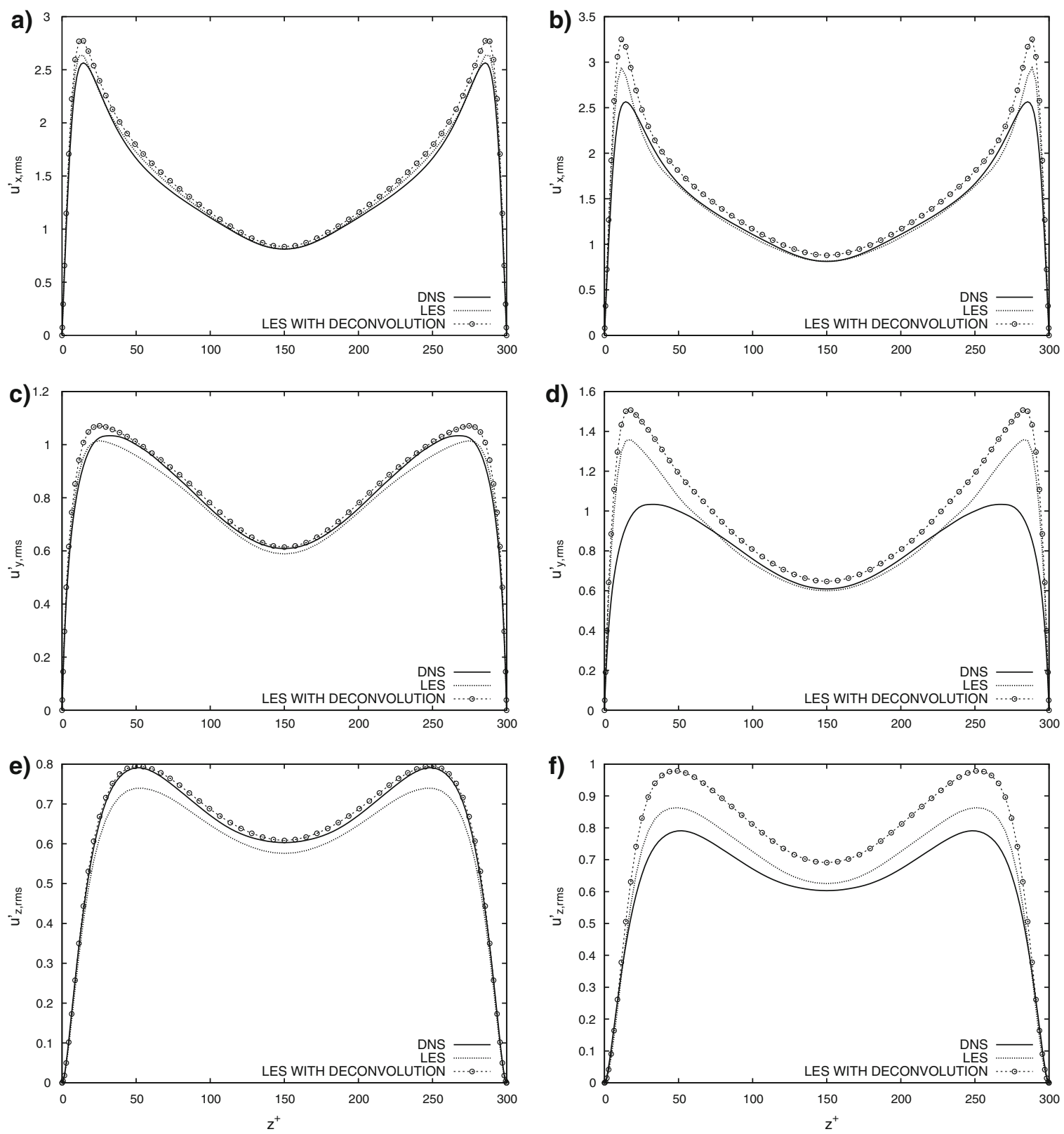

Fig. 12 RMS of fluid velocity fluctuations: comparison between DNS (solid line), LES (dotted line) and LES with closure model for the particles based on approximate deconvolution (symbols). Panels: a, b streamwise RMS; c, d spanwise RMS; e, f wall-normal RMS. Left-hand panels are relative to the fine LES; right-hand panels are relative to the coarse LES

A possible explanation for such behavior can be given by looking at the velocity signals reconstructed through fractal interpolation. Consider for instance Fig. 11, where the instantaneous DNS realization of the streamwise fluid velocity (solid line, bottom panel) previously shown in Fig. 2 is compared against an analogous streamwise velocity realization sampled from one reconstructed LES flow field (open circles, top panel). The black circles in the top panel identify the velocity values, known at the nodes of the coarse LES grid, that fractal interpolation uses as input to reconstruct the velocity signal on the DNS-like grid. When three subsequent black circles are aligned or nearly aligned, the intermediate triplets of open circles generated by fractal interpolation (like those highlighted in the figure) are also aligned or nearly aligned: the reconstructed velocity signals will be less noisy and will display lower fluctuations with respect to the coarse-grained one. Thus, starting from such LES velocity signals, the fractal interpolation technique (at least in its present implementation) is not 

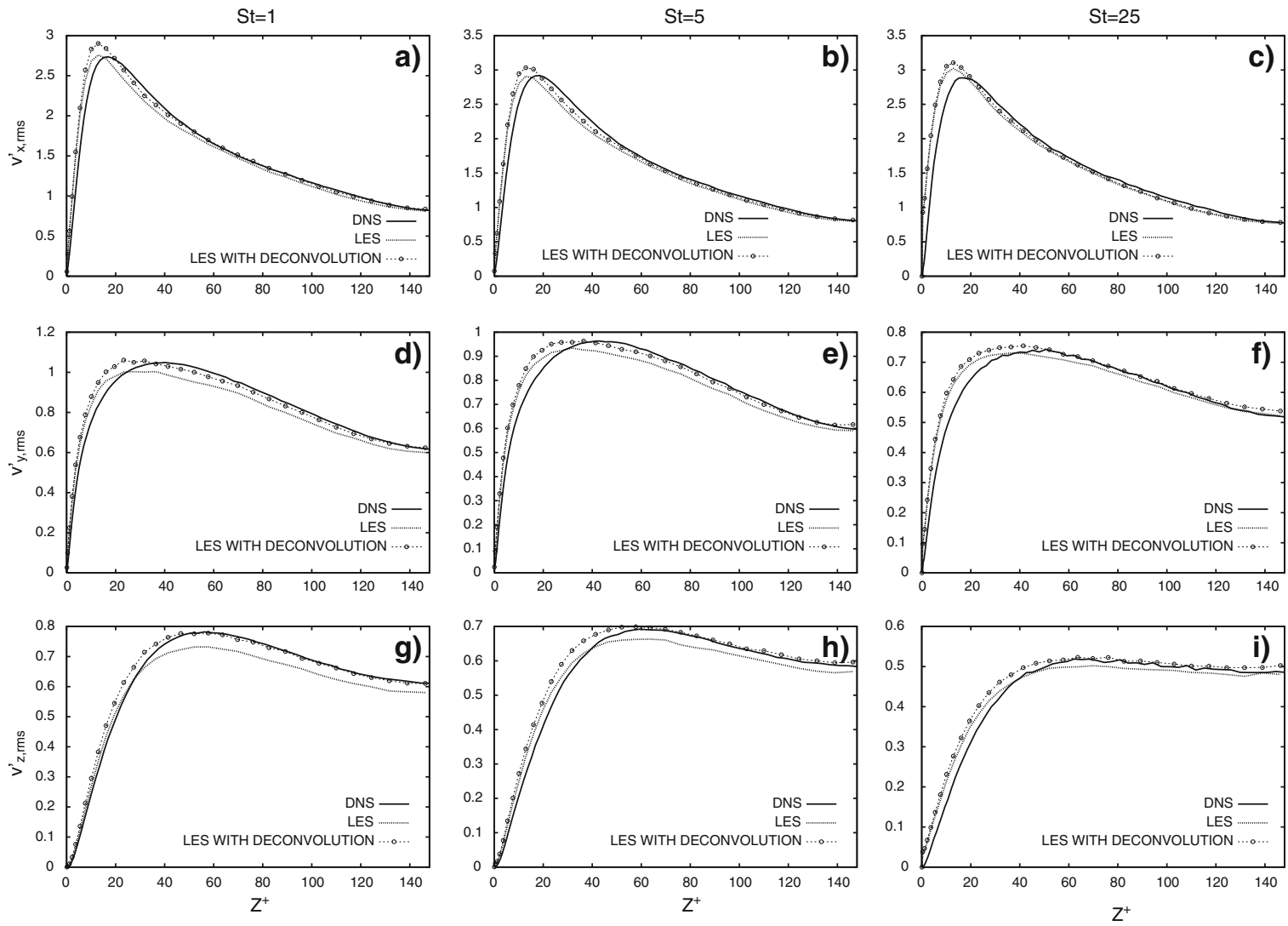

Fig. 13 RMS of particle velocity fluctuations: comparison between DNS (solid line), fine LES (dotted line) and fine LES with closure model for the particles based on approximate deconvolution (symbols). Panels: a-c streamwise RMS; d-f spanwise RMS; g-i wall-normal RMS. Columns: $S t=1$ (left); St $=5$ (center); $S t=25$ (right)

able to recover the amount of SGS turbulent kinetic energy for the fluid velocity field and it does not recover the velocity fluctuations of the two phases correctly.

\subsection{Results with SGS closure model based on approximate deconvolution}

To conclude the analysis of statistical results, we analyze now the effects of approximate deconvolution applied to the LES fluid velocity fields. Figure 12 compares the RMS values of the fluid velocity components obtained from LES and those of the fluid velocity components after deconvolution with the DNS reference values. As previously observed, the LES velocity fields obtained on the fine grid (Fig. 12a, c, e) without deconvolution are characterized by a fluctuation level in good agreement with the DNS one, except for the wall-normal component, for which LES noticeably underestimates the RMS values (Fig. 12e). As discussed in [17], this could lead to a wrong prediction of turbophoresis and, in turn, to a significant underestimation of wall particle concentration. As expected, approximate deconvolution leads to an increase of the fluid velocity RMS with respect to standard LES. For the wall-normal component the correct RMS level is recovered (Fig. 12e). However, for the remaining components the deconvolved fluid velocity field is characterized by a slight overshoot of fluctuations. As previously discussed, in the coarse LES case the LES fluid velocity field is already characterized by quite large RMS values (Fig. 12b, d, f). Approximate deconvolution increases further the fluid velocity fluctuations and, therefore, tends to amplify the disagreement with DNS.

Figures 13 and 14 show the RMS values of the particle velocity components. Considerations similar to those previously made for the RMS of the fluid velocity can be drawn. In particular, for fine LES (Fig. 13), approximate deconvolution tends to improve the agreement with DNS for the wall-normal particle velocity component, while it leads to a slight overestimation for the other components. Conversely, in coarse LES 

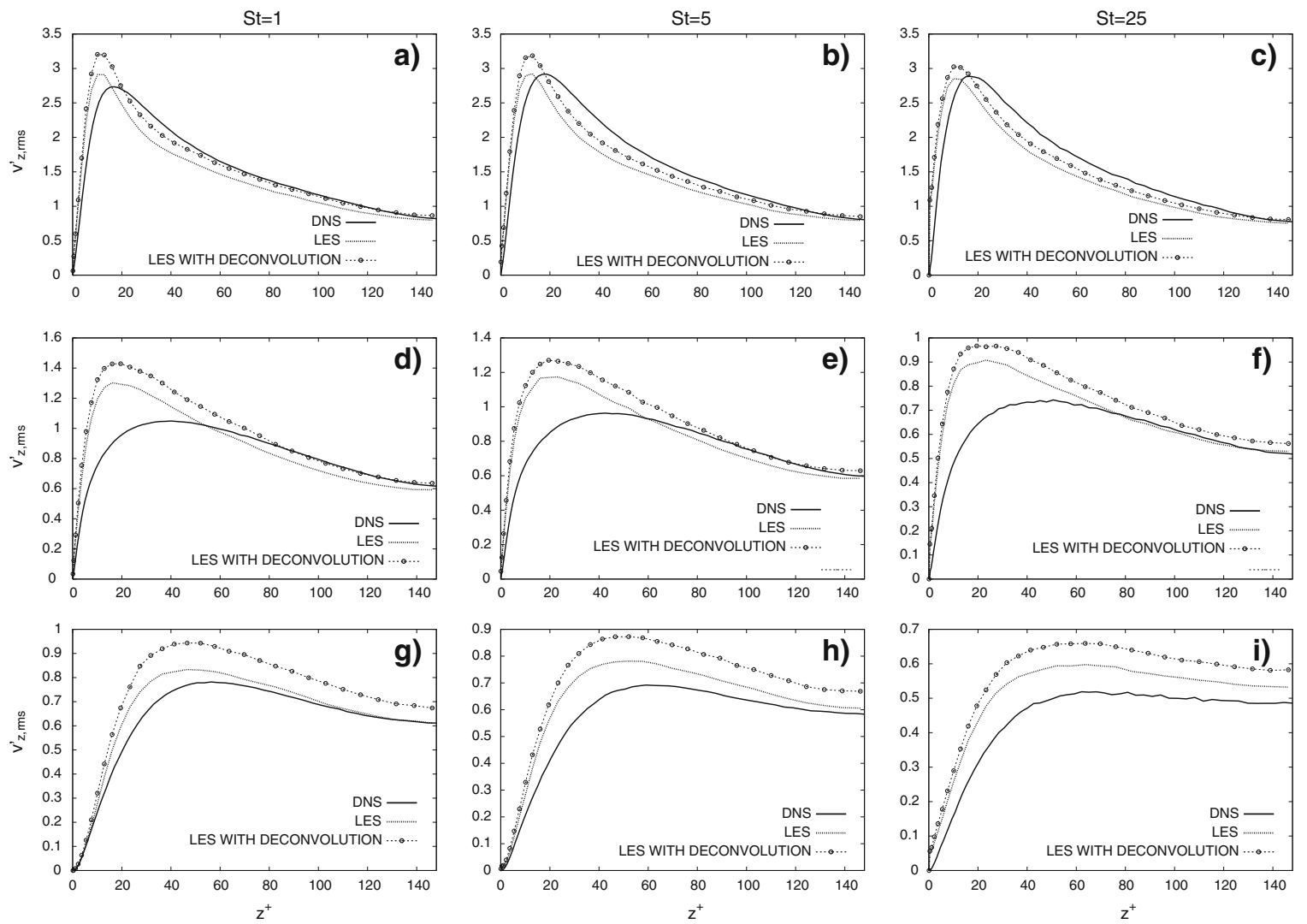

Fig. 14 RMS of particle velocity fluctuations: comparison between DNS (solid line), coarse LES (dotted line) and coarse LES with closure model for the particles based on approximate deconvolution (symbols). Panels: a-c streamwise RMS; d-f spanwise RMS; g-i wall-normal RMS. Left-hand column $S t=1$, central column $S t=5$, right-hand column $S t=25$

(Fig. 14), the RMS values are overestimated for all the considered particle sets and all the velocity components: When approximate deconvolution is applied to the fluid velocity fields, overestimation becomes larger.

Finally, Fig. 15 shows the instantaneous particle concentration profiles. It can be seen that, in all cases, approximate deconvolution improves the agreement with DNS [17,26]. Prediction of the deposition velocity (results not shown) also improves. This is not surprising in the fine LES case, since approximate deconvolution proved its capability to recover the correct level of fluctuations for the wall-normal velocity component of both phases (see Figs. 12e and 13g, h, i). As previously mentioned and as discussed in [17], this is a key issue to have a good prediction of turbophoresis. In the coarse LES case, this improvement seems to be rather due to a compensation of errors, since the RMS values of both fluid and particle wall-normal component are largely overestimated with approximate deconvolution. It can be argued that this overestimation of velocity fluctuations tends to compensate the inadequate description in LES of the physical mechanism leading to turbophoresis, probably due to inaccurate rendering of the interaction between wall turbulence structures and particles (see Sect. 1). However, this point needs further investigation.

\section{Conclusions and future developments}

In this paper, the capabilities of two different SGS closure models for the equations of particle motion are investigated in Lagrangian tracking of particles one-way coupled with LES fluid velocity fields. These models are based on two different techniques, namely fractal interpolation and approximate deconvolution of the filtering operator applied in LES. Both techniques are aimed at reconstructing at least part of the velocity field fluctuations lost in LES (SGS fluctuations) in order to reintroduce such fluctuations into the equations of particle motion.

The candidate SGS closures have been appraised in Lagrangian tracking of particles using the fluid velocity fields obtained in two independent large-eddy simulations of turbulent channel flow, each characterized by 

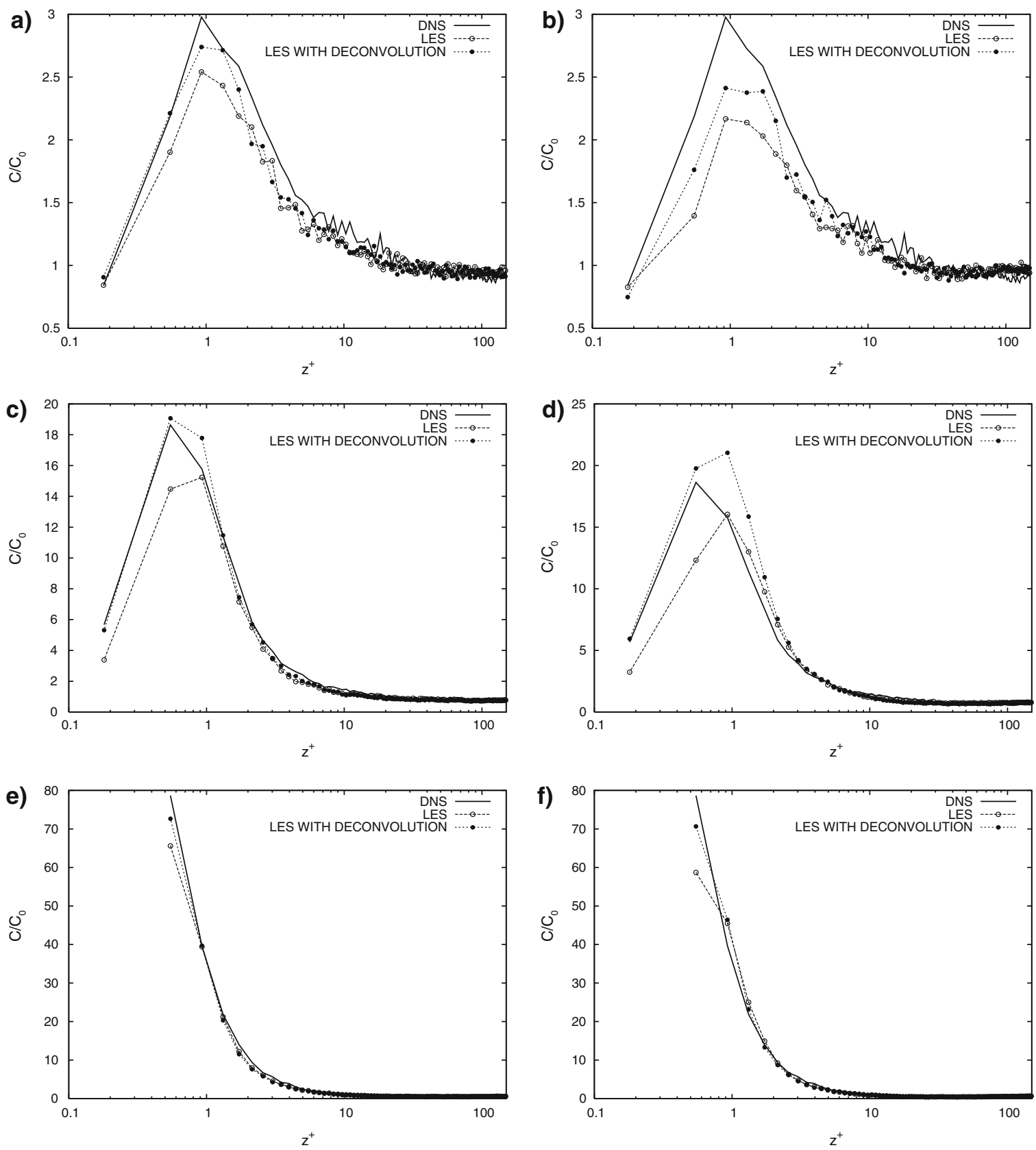

Fig. 15 Effect of the closure model on particle concentration: comparison between DNS (solid line), LES (open circles) and LES with closure model based on approximate deconvolution (black circles). Panels: a, b $S t=1 ; \mathbf{c}, \mathbf{d} S t=5 ; \mathbf{e}, \mathbf{f} S t=25$. Left-hand panels are relative to the fine LES; right-hand panels are relative to the coarse LES

different grid resolutions. Three sets of particles having different inertia (with Stokes numbers equal to 1, 5 and 25 , respectively) have been considered. The accuracy in the prediction of the particle velocity statistics and near wall accumulation is assessed through direct comparison against DNS data.

Results obtained for LES with no closure model in the particle equations indicate that, in the fine LES case, the level of fluid velocity fluctuations is in good agreement with DNS (as expected for a well-resolved LES) except for a noticeable underestimation of the fluctuations of the wall-normal velocity component. Conversely, in the coarse LES, the RMS of all velocity components are largely overestimated and this is plausibly an effect of the modeling error in the LES equations for the fluid phase. The same considerations can be made for the RMS of the particle velocity components over the entire range of Stokes numbers considered in this study. In spite of 
these differences in the velocity statistics, the near-wall particle concentration is significantly underestimated in both fine and coarse LES, and this underestimation is larger for the coarse-grained simulation. This is a first indication that the satisfactory behavior of LES in reproducing particle velocity statistics is counterbalanced by the inaccurate representation of local segregation phenomena, and that reintroducing the correct amount of fluid and particle fluctuations may not be enough to have a correct prediction of turbophoresis.

As far as the effects of the SGS closures for the particle equations are considered, 2D fractal interpolation appears to be inefficient in reintroducing the fluid velocity fluctuations, at least for the low Reynolds number considered here. Indeed, for both LES grids considered in this study the velocity signals varies rather smoothly in space and this makes the fractal interpolation procedure inefficient (there is no fractal form to be copied). Consequently, no positive impact has been found in the prediction of particle statistics and concentration. As possible future development, it would be interesting to evaluate the impact of performing a fully $3 \mathrm{D}$ fractal interpolation, extending it to the wall-normal direction, and to apply the procedure to flows at higher Reynolds numbers, in which a wider range of interacting scales is contained.

Conversely, approximate deconvolution indeed tends to increase the level of fluctuations of the LES fluid velocity. In the fine LES with approximate deconvolution, a good agreement is obtained with DNS for the RMS of both fluid and particle velocities, also for the wall-normal component. This leads to a significant improvement in the prediction of near-wall particle concentration. For the coarse LES, the overestimation of the fluid and particle velocity fluctuations tends to be amplified by approximate deconvolution and the agreement with DNS is further deteriorated. Nevertheless, an improvement in the prediction of the wall-normal particle concentration is obtained. This improved agreement might be due to error compensation, i.e. the overestimation of velocity fluctuations tends to compensate the inability of LES to provide accurate representation of the physical mechanism leading to turbophoresis, namely the interaction between wall turbulence structures and particles.

The results shown in this paper indicate that reintroduction of the correct amount of velocity fluctuations does not warrant accurate prediction of near-wall accumulation and suggest that additional information on the flow field at the sub-grid level is required [14]. Our feeling is that accurate sub-grid closure models for particles require information also proportional to the higher order moments of the velocity fluctuations. In this case, non-Gaussian stochastic Lagrangian models based on Langevin-type equations might be more suitable [37].

\section{References}

1. Wang, L.P., Riley, M.R.: Settling velocity and concentration distribution of heavy particles in homogeneous isotropic turbulence. J. Fluid Mech. 256, 27-68 (1993)

2. Bec, J., Biferale, L., Cencini, M., Lanotte, A., Musacchio, S., Toschi, F.: Heavy particle concentration in turbulence at dissipative and inertial scales. Phys. Rev. Lett. 98, 084502 (2007)

3. Eaton, J.K., Fessler, J.R.: Preferential concentration of particles by turbulence. Int. J. Multiphase Flow 20, 169-209 (1994)

4. Rouson, D.W., Eaton, J.K.: On the preferential concentration of solid particles in turbulent channel flow. J. Fluid Mech. 428, 149-169 (2001)

5. Brooke, J.W., Kontomaris, K., Hanratty, T.J., McLaughlin, J.B.: Turbulent deposition and trapping of aerosols at a wall. Phys. Fluids A 4, 825-834 (1992)

6. Marchioli, C., Soldati, A.: Mechanisms for particle transfer and segregation in turbulent boundary layer. J. Fluid Mech. 468, 283-315 (2002)

7. García, M., Lopez, F., Niño, Y.: Characterization of near-bed coherent structures in turbulent open channel flow using synchronized high-speed video and hot-film measurements. Exp. Fluids 19, 16-28 (1995)

8. Kaftori, D., Hetsroni, G., Banerjee, S.: Particle behavior in the turbulent boundary layer. I. Motion, deposition, and entrainment. Phys. Fluids 7, 1095-1106 (1995)

9. Adrian, R.J.: Hairpin vortex organization in wall turbulence. Phys. Fluids 19, 041301 (2007)

10. Soldati, A.: Particles turbulence interactions in boundary layers. Z. Angew. Math. Mech. 85, 683-699 (2005)

11. Caporaloni, M., Tampieri, F., Trombetti, F., Vittori, O.: Transfer of particles in nonisotropic air turbulence. J. Atmos. Sci. 32, 565-568 (1975)

12. Reeks, M.W.: The transport of discrete particles in inhomogeneous turbulence. J. Aerosol Sci. 14, 729-739 (1983)

13. Young, J.B., Hanratty, T.J.: Optical studies on the turbulent motion of solid particles in a pipe flow. J. Fluid Mech. 231, 665-668 (1991)

14. Marchioli, C., Salvetti, M.V., Soldati, A.: Some issues concerning Large-Eddy Simulation of inertial particle dispersion in turbulent bounded flows. Phys. Fluids 20, 040603 (2008)

15. Février, P., Simonin, O., Squires, K.D.: Partitioning of particle velocities in gas-solid turbulent flows into a continuous field and a spatially-uncorrelated random distribution: theoretical formalism and numerical study. J. Fluid Mech. 533, 1-46 (2005)

16. Kuerten, J.G.M.: Subgrid modeling in particle-laden channel flow. Phys. Fluids 18, 025108 (2006)

17. Kuerten, J.G.M., Vreman, A.W.: Can turbophoresis be predicted by large-eddy simulation? Phys. Fluids 17, 011701 (2005)

18. Wang, Q., Squires, K.D.: Large eddy simulation of particle deposition in a vertical turbulent channel flow. Int. J. Multiphase Flow 22, 667-683 (1996) 
19. Tian, L., Ahmadi, G.: Particle deposition in turbulent duct flows-comparisons of different model predictions. J. Aerosol Sci. 38, 377-397 (2007)

20. Marchioli, C., Giusti, A., Salvetti, M.V., Soldati, A.: Direct numerical simulation of particle wall transfer and deposition in upward turbulent pipe flow. Int. J. Multiphase Flow 29, 1017-1038 (2003)

21. Uijttewaal, W.S.J., Oliemans, R.W.A.: Particle dispersion and deposition in direct numerical and large eddy simulations of vertical pipe flows. Phys. Fluids 8, 2590-2604 (1996)

22. Fede, P., Simonin, O.: Numerical study of the subgrid fluid turbulence effects on the statistics of heavy colliding particles. Phys. Fluids 18, 045103 (2006)

23. Reza Keshevarzi, A., Nagi Ziaei, A., Homayoun, E., Shirvani, A.: Fractal-Markovian scaling of turbulent bursting processes in open channel flows. Chaos Solitons Fractals 25, 307-318 (2005)

24. Scotti, A., Meneveau, C.: A fractal model for large eddy simulation of turbulent flow. Phys. D 127, 198-232 (1999)

25. Shotorban, B., Mashayek, F.: Modeling subgrid-scale effetcs on particles by approximate deconvolution. Phys. Fluids 17, 081701 (2005)

26. Shotorban, B., Zhang, K.K.Q., Mashayek, F.: Improvement of particle concentration prediction by defiltering. Int. J. Heat Mass Tran. 50, 3728-3739 (2007)

27. Germano, M., Piomelli, U., Moin, P., Cabot, W.H.: A dynamic subgrid-scale eddy viscosity model. Phys. Fluids 3, 1760-1765 (1991), Erratum, Phys. Fluids 3, 3128 (1991)

28. Elghobashi, S.E., Truesdell, G.C.: Direct simulation of particle dispersion in a decaying isotropic turbulence. J. Fluid Mech. 242, 655-700 (1992)

29. Crowe, C., Sommerfeld, M., Tsuji, T.: Multiphase Flows with Droplets and Particles. CRC Press, New York (1998)

30. Lam, K., Banerjee, S.: Streak formation in a bounded turbulent-flow. Phys. Fluids A 4, 306-326 (1992)

31. Soldati, A., Banerjee, S.: Turbulence modification by large-scale organized electrohydrodynamic flows. Phys. Fluids 10, 1742-1756 (1998)

32. Marchioli, C., Picciotto, M., Soldati, A.: Particle dispersion and wall-dependent fluid scales in turbulent bounded flow: implications for local equilibrium models. J. Turbulence 27, 1-11 (2006)

33. Marchioli, C., Soldati, A., Kuerten, J.G.M., Arcen, B., Tanière, A., Goldensoph, G., Squires, K.D., Cargnelutti, M.F., Portela, L.M.: Statistics of particle dispersion in Direct Numerical Simulations of wall-bounded turbulence: results of an international collaborative benchmark test. Int. J. Multiphase Flow 34 (2008). doi:10.1016/j.ijmultiphaseflow.2008.01.009

34. Salvetti, M.V., Marchioli, C., Soldati, A.: On the closure of particle motion equations in large-eddy simulation. In: Lamballais, E., Friedrich, R., Geurts, B.J., Metais, O. (eds.) Direct and Large-Eddy Simulation, vol. 6, pp. 311-318. Springer, Netherlands (2006)

35. Stolz, P., Adams, N.A., Kleiser, L.: An approximate deconvolution model for large-eddy simulation with application to incompressible wall-bounded flows. Phys. Fluids 13, 997-1015 (2001)

36. Cousins, L.B., Hewitt, G.F.: Liquid phase mass transfer in annular two-phase flow. UKAEA Report, AERE-R 5657 (1968)

37. Guingo, M., Minier, J.-P.: A stochastic model of coherent structures for particle deposition in turbulent flows. Phys. Fluids 20, 053303 (2008) 